\title{
Monitoramento da Integridade Estrutural de uma laje de concreto utilizando transformadas de wavelet
}

\section{Structural Health Monitoring of concrete slab using wavelet transforms}

\author{
${ }^{1}$ Pedro José Trindade Campos, ${ }^{2}$ Edson Hideki Koroishi, ${ }^{3}$ Albert Willian Faria \\ 1Engenheiro Civil pela Universidade Federal do Triângulo Mineiro (pedrotrindade.engcivil@ gmail.com) \\ ${ }^{2}$ Doutor em Engenharia Mecânica, Professor Adjunto do Departamento de Engenharia Mecânica da \\ Universidade Tecnológica Federal do Paraná (edsonh @utfpr.edu.br) \\ ${ }^{3}$ Doutor em Engenharia Mecânica, Professor Associado do Departamento de Engenharia Civil da \\ Universidade Federal do Triângulo Mineiro (albert.faria@uftm.edu.br)
}

\begin{abstract}
RESUMO: A presente contribuição utiliza uma metodologia para localizar danos em um sistema estrutural do tipo laje de concreto empregando transformadas de Wavelet. Esse sistema estrutural é modelado numericamente empregando o Método dos Elementos Finitos (MEF). O sinal da deflexão vertical da laje danificada é utilizado na localização da posição do dano empregando a transformada discreta de wavelet (TDW). As wavelets mães estudadas foram a: Daubechies, Symlets e ReverseBior. O tipo de dano implementado é dado sob a forma de perda localizada da rigidez do concreto da laje utilizando uma variável escalar de dano $D$. O sinal da deflexão, obtido na análise estática da laje, é interpolado e depois ampliado empregando a regularização de Tikhonov antes de ser transformado. O elemento finito utilizado na modelagem da laje apresenta quatro nós e seis graus de liberdade por nó, sendo três translações e três rotações. A calibração dos parâmetros $\beta_{j}$ da regularização de Tikhonov mostrouse fundamental para amplificar e suavizar as perturbações nos gráficos dos coeficientes de wavelet. Além disso, todas as wavelets mães avaliadas foram capazes de identificar o dano proposto. Entretanto, quando a variável escalar de dano é maior ou igual a 0,20 , somente o sinal da estrutura na condição danificada foi necessário para detectar a posição do dano. Já, para valores de dano menores que 0,20 , foi necessário o emprego do sinal da diferença, obtido entre as condições da estrutura sem e com dano.
\end{abstract}

Palavras-chave: Monitoramento da integridade estrutural. Método dos elementos finitos. Wavelet transform. Regularização de Tikhonov.

ABSTRACT: This paper describes the methodology to locate the damage area on the concrete slab structure. The damaged structure is numerically simulated using the Finite Element Method (FEM). The vertical displacement signal has obtained is using for location of the damage area position employing the Discrete Wavelet Transform (DWT). The mother wavelets studied were: Daubechies, Symlets and ReverseBior. The damage on the slab is numerically simulated as one stiffness reduction in the concrete and the displacement signal obtained from the static analysis is interpolated and then amplified by means of the Tikhonov regularization. The finite element is quadrilateral with corner nodes only and each node has six degrees of freedom per node, i.e., three translations and three rotations. The calibration of Tikhonov regularization parameters $\beta_{j}$ is important to amplify and smooth the perturbations of wavelet coefficients graphs. In addition, all the mother wavelets available in this paper have being able to identify the damage. Results suggest when the damage reduces the concrete modulus of elasticity more than $20 \%$ it is only necessary the knowledge of signal of damage structure to detect the damaged area position. For the smaller damage values, it is required to know the different between the displacement signals about the undamaged and damaged structure.

Keywords: Structural Health Monitoring. Finite Element Method. Wavelet transform. Tikhonov regularization.

\section{INTRODUÇÃO}

O Monitoramento de Integridade Estrutural (Structural Health Monitoring - SHM) é uma técnica amplamente estudada no meio científico em vista à detecção e avaliação de falhas em sistemas estruturais fabricados em materiais diversos. O SHM pode substituir com eficiência a manutenção corretiva pela preditiva e preventiva, mais baratas e que diminuem as manutenções fora de época que atrapalham a produção nos grandes setores industriais, tais como nos setores petroquímico, naval e aeroespacial (Maio, 2011), e, mais recentemente na construção civil. 
Uma técnica eficiente para identificação do dano deve ser incorporada ao sistema estrutural monitorado, visto que, alterações (trincas, fissuras e outras) nas respostas estática ou dinâmica da estrutura, em sua fase inicial, são visualmente imperceptíveis. Neste sentido, segundo Yan e Yam (2002), a Transformada de Wavelet (TW), tradicionalmente empregada no tratamento de imagens e sinais, vem recebendo bastante interesse nos últimos anos no SHM de estruturas diversas, devido a sua fácil implementação numérica, por fornecer representações do sinal no domínio do tempo e da frequência e por permitir a escolha da função de transformação (wavelet mãe) que melhor valorize ou maximize diferentes características do sinal (singularidades e descontinuidades) a ser analisado.

Uma das grandes vantagens do uso da TW no SHM de sistemas estruturais é que qualquer sistema de aquisição de dados e um simples microcomputador portátil podem ser empregados para monitorar, ao longo do tempo ou da frequência, o sistema, eliminando-se assim a necessidade do uso de equipamentos caros e pesados (tais como os impedanciômetros). Além disso, essa técnica de monitoramento pode ser incorporada a análise dos dados oriundos do Ensaio de Prova de Carga (normatizado pelas diretrizes da NBR9607 (ABNT, 1986)) que é comumente empregado nos laudos técnicos para a avaliação do Estado Limite Último (ELU) e do Estado Limite de Serviço (ELS) de estruturas de concreto.

O Estado Limite Último (ELU) é relacionado ao colapso, ou a qualquer outra forma de ruína estrutural, que determine a paralisação do uso da estrutura. A segurança das estruturas de concreto deve sempre ser verificada em relação aos seguintes ELU (Camacho, 2015): perda do equilíbrio da estrutura, admitida como corpo rígido; esgotamento da capacidade resistente da estrutura, no seu todo ou em parte, devido às solicitações normais e tangenciais; esgotamento da capacidade resistente da estrutura, no seu todo ou em parte, considerando os efeitos de segunda ordem; solicitações dinâmicas e casos especiais. Já o Estado Limite de Serviço (ELS) é, segundo Camacho (2015), aquele que corresponde à impossibilidade do uso normal da estrutura, estando relacionado: à durabilidade, à aparência, ao conforto do usuário e a boa utilização funcional da mesma, seja em relação aos usuários, seja em relação as máquinas e equipamentos utilizados. E, pode se originar de uma das seguintes causas: formação de fissuras; abertura de fissuras; deformações excessivas; vibrações excessivas e outros casos especiais.

Este artigo interessa-se no SHM de uma laje irregular (no formato em "L") de concreto, danificada em uma área arbitrária, empregando a TW ao sinal da deflexão vertical, obtida via análise estática desse sistema estrutural. A grande maioria dos trabalhos disponíveis na literatura científica sobre o SHM através do uso da TW focam-se no estudo de outros tipos de sistemas estruturais de geometria mais simples, tais como: vigas, placas retangulares e treliças, além disso, empregando condições de contorno (apoios) mais simples que os propostos nesse trabalho.

A modelagem numérica da laje de concreto é feita via Método dos Elementos Finitos (MEF), que segundo Bathe (2014), tem por princípio discretizar um modelo físico contínuo, através da sua divisão em subdomínios com forma geométrica simples, chamados elementos finitos. Além de ser uma ferramenta de engenharia madura e cujas potencialidades e limitações são amplamente conhecidas e/ou estudadas desde 1950, esse método tem-se mostrado o mais adequado para a modelagem de estruturas diversas em virtude de sua flexibilidade de modelagem (variando-se com facilidade a geometria, as condições de contorno e os carregamentos do modelo numérico implementado), relativa facilidade de implementação numérica nos mais diversos tipos de problemas da Engenharia e devido a possibilidade de substituírem ensaios experimentais destrutivos.

O uso da TW no SHM de diversos tipos de sistemas estruturais modelados numericamente através do MEF tem mobilizado nas últimas décadas diversos pesquisadores tais como: Ovanesova e Suárez (2004) no estudo de estruturas danificadas do tipo viga e pórtico utilizando as TW discreta e contínua por meio das wavelets mães Haar e Bior (biorthogonal); 
Yang et al. (2011) no estudo experimental e numérico de placas de alumínio danificadas (dotadas de enrijecedores) através do uso da TW discreta do tipo Packet; Janeliukstis et al. (2016) na detecção do dano em uma placa de alumínio retangular utilizando a TW contínua Pet Hat; e, Faria et al. (2017) no estudo da detecção do dano em placas de materiais compósitos laminados danificadas utilizando as TW direta e contínua. No entanto, poucos são os trabalhos que empregam as TW no estudo de lajes de concreto, especialmente com formato geométrico irregular, comumente encontradas na área da construção civil. Dessa forma, esse artigo procura também incrementar os estudos numéricos e a revisão bibliográfica nacional sobre a temática do uso da TW no SHM de estruturas de concreto.

A localização da região danificada em estruturas é importante em vista à otimização do posicionamento e da quantidade de reforço estrutural a ser utilizado na estrutura danificada (através, por exemplo, do emprego de mantas de material compósito polimérico), de forma a minimizar os efeitos do dano na estrutura avaliada e de propiciar também economia de tempo de uso e de custo de implantação do sistema de reforço.

O mecanismo de dano proposto nesse artigo é dado na forma de perda de rigidez do concreto em uma determinada região arbitrária da laje, que pode ocorrer, por exemplo, durante a fase de sua execução por diversos fatores, dentre eles: problema na dosagem do concreto a ser lançado; excesso ou falta de água durante a cura do concreto e devido ao emprego do concreto (em determinadas regiões da laje) após o tempo inicial das reações químicas de cristalização do cimento que culminam no endurecimento e na solidificação da mistura (pega do concreto). Esse tipo de danificação em lajes de concreto tem levado a inúmeros trabalhos de consultoria técnica em virtude do aparecimento de deformações/vibrações excessivas (ELS), pela abertura de fissuras nas estruturas de concreto (ELS) ou devido a perda da capacidade resistente da estrutura de concreto (ELU), e que devem ser avaliados conforme diretrizes fornecidas na NBR6118 (ABNT, 2014).

\section{MATERIAIS E MÉTODOS}

De acordo com Addison (2017), o estudo da TW se iniciou na metade da década de 80, devido à necessidade de estudar sinais $(s)$ advindos de abalos sísmicos e terremotos (sinais não estacionários), de maneira mais precisa do que era feita anteriormente através do uso da Transformada de Fourier.

Segundo Stark (2005) e Addison (2017), a TW contínua (também chamada transformada contínua de wavelet - TCW) pode ser definida matematicamente como o produto interno das funções $s(t)$ e $\psi_{a, b}^{*}(t)$ segundo a Eq. 1:

$$
W(a, b)=\int_{-\infty}^{\infty} s(t) \psi_{a, b}^{*}(t) d t=\frac{1}{\sqrt{a}} \int_{-\infty}^{\infty} s(t) \psi\left(\frac{t-b}{a}\right) d t=\left\langle s, \psi_{a, b}^{*}\right\rangle
$$

sendo: $s(t)$ sinal a ser decomposto; $\psi^{*}$ denota o complexo conjugado de $\psi$ definido pelo parâmetro de escala $(a)$, que altera a escala da wavelet formada pela função $\psi(t)$, e pelo parâmetro de translação $(b)$, que representa a distância com que a função $\psi(t)$ foi transladada no eixo $\mathrm{t}(b \in \boldsymbol{R}, a>0)$, e $\sqrt{a}^{-1}$ é um fator de normalização. A função $\psi_{1,0}(t)$ é chamada de wavelet mãe, enquanto as outras funções $\psi_{a, b}(t)$ são chamadas de wavelets filhas.

Em um domínio discreto, os parâmetros de escala e de translação são discretizados como: $a=a_{0}^{m}$ e $b=n b_{0} a_{0}^{m}$, e as wavelets analisadas podem ser discretizadas sob a forma (Addison, 2017): 
Campos; P. J. T.; Koroishi, E. H.; Faria, A. W.; Monitoramento da integridade estrutural de uma laje de concreto utilizando transformadas de wavelet. E\&S - Engineering and Science, 2020, 10:1.

$$
\psi_{m, n}(t)=\frac{1}{a_{0}^{m / 2}} \psi^{*}\left(\frac{t-n b_{0} a_{0}^{m}}{a_{0}^{m}}\right)
$$

onde $m$ e $n$ são valores inteiros que controlam respectivamente a dilatação e a translação, $a_{0}$ é um parâmetro fixo de dilatação maior que a unidade e $b_{0}$ é um parâmetro de localização que deve ser maior que 0 . A situação mais comum é dada assumindo que $a_{0}=2^{1 / \bar{v}} \operatorname{com} \bar{v}$ um valor inteiro, e $b_{0}=1$ (Addison, 2017).

A Transfomada de Wavelet de um sinal contínuo $s(t)$ usando as wavelets discretas da Eq. 2 é chamada transformada discreta de wavelet (TDW), dada sob a forma (Addison, 2017):

$$
T_{m, n}=\int_{-\infty}^{+\infty} s(t) \frac{1}{a_{0}^{m / 2}} \psi^{*}\left(a_{0}^{-m} t-n b_{0}\right) d t=\left\langle s, \psi_{m, n}\right\rangle
$$

sendo $T_{m, n}$ valores discretos dados em um plano bidimensional escala-localização de índices $m$ e $n$ conhecidos como coeficientes de wavelet $(\mathrm{CW})$.

Há diversos tipos de wavelets mães que podem ser empregadas na TW e dentre elas as mais usuais são a: Meyer (dmey), Haar (haar), Daubechies (db), Symlets (sym), Coiflets (coif), BiorSplines (bior) e a ReverseBior (rbior), cada qual apresentando domínios específicos de atuação. Normalmente as wavelets mães constituintes de uma mesma família de wavelets são escritas utilizando um número $\mathrm{N}$ escrito após o seu nome (como por exemplo, dbN) que indica a ordem da wavelet.

A metodologia proposta nesse artigo utiliza conjuntamente as plataformas computacionais ANSYS e Matlab, empregadas respectivamente na modelagem numérica (via MEF) da laje de concreto e para a localização (via TW) da posição do dano. O sinal $\overline{\boldsymbol{s}}$, ou seja, o deslocamento vertical (deflexão) nodal sofrido pela estrutura na condição danificada (e, se necessário, também na condição não danificada) é obtido realizando-se a análise estática do modelo numérico implementado via MEF no ANSYS.

Antes de ser transformado, o sinal estático é interpolado utilizando «splines» cúbicas, para incrementar o número de dados do sinal, e também é submetido a técnica de regularização de Tikhonov, para amplificar e suavizar as perturbações nos gráficos dos $\mathrm{CW}$ pela presença do dano, resultando no sinal $s$.

No processo de interpolação polinomial por partes o sinal $\overline{\boldsymbol{s}}$, em cada subintervalo $\left[x_{k-1}, x_{k}\right]$, com $k=1,2 \ldots, n$, pode ser aproximado por meio de um polinômio de terceiro grau do tipo (Galvão e Nunes, 2021; Chapra, 2013):

$$
\bar{s}_{k}(x)=a_{k}+b_{k}\left(x-x_{k}\right)+c_{k}\left(x-x_{k}\right)^{2}+d_{k}\left(x-x_{k}\right)^{3}
$$

com coeficientes: $a_{k}=f\left(x_{k}\right)=f_{k}, b_{k}=\left(f_{k}-f_{k-1}\right) / h_{k}+\left(2 h_{k} g_{k}+g_{k-1} h_{k}\right) / 6, c_{k}=g_{k} / 2$ e $d_{k}=\left(g_{k}-g_{k-1}\right) / 6 h_{k}$, com $h_{k}=x_{k}-x_{k-1}$. Já, os valores de $g_{k}$ informados são obtidos da solução do sistema linear (Galvão e Nunes, 2021):

$$
h_{k} g_{k-1}+2\left(h_{k}+h_{k+1}\right) g_{k}+h_{k+1} g_{k+1}=6\left(h_{k+1}^{-1}\left(f_{k+1}-f_{k}\right)-h_{k}^{-1}\left(f_{k}-f_{k-1}\right)\right)
$$

com $(n-1)$ equações (ou seja: $k=1,2,3 \ldots, n$-1) e $(n+1)$ incógnitas: $g_{0}, g_{1}, \ldots, g_{n}$. Adotando $g_{0}=$ $g_{n}=0$ tem-se as chamadas «splines» cúbicos naturais e o sistema linear expressado na Eq. 5 torna-se determinado. 
No Matlab, a rotina nativa chamada «splines» é usada neste trabalho para a interpolação cúbica do sinal $\overline{\boldsymbol{s}}$, empregando a linha de comando « yy $=$ spline $(\mathrm{x}, \overline{\boldsymbol{s}}, \mathrm{xq})$ », sendo x e $\overline{\boldsymbol{s}}$ respectivamente vetores contendo a posição do nó de captura da resposta e os valores do deslocamento vertical em cada um desses nós. Já xq é um vetor que contém os pontos de interesse a serem interpolados utilizado na determinação do vetor yy (dos resultados da interpolação). Neste trabalho, o vetor xq é criado a partir da definição de um incremento de pontos $\Delta \mathrm{x}$ no vetor $\mathrm{x}$.

A regularização de Tikhonov por sua vez pode ser expressa em diferenças finitas sob a forma da expressão (Beck et al. 1985; Costa Silva, 2015):

$$
\mathbf{s}=\beta_{0} \sum_{i=1}^{\rho}\left(s_{i}^{n}\right)^{2}+\beta_{1} \sum_{i=1}^{\rho}\left(s_{i}^{n}-s_{i}^{n-1}\right)^{2}-\beta_{2} \sum_{i=1}^{\rho}\left(s_{i}^{n}-2 s_{i}^{n-1}+s_{i}^{n-2}\right)^{2}
$$

sendo $\beta_{j}$ os $j(j=0: 2)$ parâmetros de regularização, $s_{i}$ são as componentes do vetor a ser regularizado e $n$ é o número de iteração. Nesse artigo, os parâmetros de regularização utilizados são adotados iguais, ou seja: $\beta_{0}=\beta_{1}=\beta_{2}$, e suas magnitudes são indicadas nas simulações numéricas realizadas, escolhidas de maneira a ampliar os valores dos CW na região danificada.

No Matlab, o sinal $s$ fornecido da Eq. 6 é transformado via TDW e analisado através da obtenção dos coeficientes de wavelet $(\mathrm{CW})$. Nesse trabalho são utilizadas as wavelets mães: Daubechies (db), Symlets (sym) e a ReverseBior (rbior), todas já se encontram implementadas na caixa de ferramentas (toolbox) «wavemenu» do Matlab.

O mecanismo de dano é inserido na estrutura analisada pela adoção de uma variável escalar de dano $(D)$, assumida invariável no tempo $(t)$, através da redução do módulo de Young inicial $(E)$ do concreto constituinte da laje na condição não danificada, utilizando a expressão: $\tilde{E}=(1-D) E . \tilde{E}$ é definido nesse trabalho como sendo o módulo de elasticidade do material na condição danificada. Nessa expressão, quando $D=0$ tem-se o estado não danificado da estrutura e quando $D>0$, tem-se o estado dito danificado.

A equação global do movimento do sistema estrutural danificado, obtida via MEF, pode ser expressa sob a forma:

$$
\boldsymbol{K}_{g}(D) \boldsymbol{u}(D)=\boldsymbol{f}_{g}
$$

onde: $\boldsymbol{u}(D)$ é o vetor dos deslocamentos nodais dependente da variável escalar de dano $D, \boldsymbol{f}_{g}$ é o vetor das forças globais e a matriz $\boldsymbol{K}_{g}(D)$ é a matriz de rigidez global modificada pela variável $D$ devido a diminuição direta do módulo de elasticidade inicial $(E)$ do material da estrutura. O vetor $\boldsymbol{u}(D)$ é obtido (após a aplicação das condições de contorno, ou seja, restrição dos deslocamentos dos apoios da laje) no módulo «solver» do software ANSYS pela inversão da matriz de rigidez global multiplicada pelo vetor das forças globais, ou seja, $\boldsymbol{u}(D)=$ $\boldsymbol{K}_{g}(D)^{-1} \boldsymbol{f}_{g}$. O vetor $\boldsymbol{u}(D)$ contém o sinal estático $s$ a ser transformado pela TW, após ser interpolado e regularizado.

\section{RESULTADOS E DISCUSSÕES}

A laje estudada nesse artigo, proposta inicialmente no trabalho de Hecke (2012), é ilustrada na figura 1(a). Ela apresenta espessura constante e igual a $0,12 \mathrm{~m}$, formato geométrico "L" invertido e é sujeita a uma pressão uniformemente distribuída, sobre toda a sua superfície, 
igual a $20.000 \mathrm{~N} / \mathrm{m}^{2}$. O concreto da laje possui módulo de elasticidade $(E)$ igual a $1500 \mathrm{MPa}$ e coeficiente de Poisson ( $v$ ) igual a 0,20.

O elemento finito utilizado para a discretização da laje de concreto é um elemento plano e linear dotado de seis graus de liberdade (gdl) por nó, sendo três translações $(u, v$ e $w)$ e três rotações $\left(\theta_{x}, \theta_{y}\right.$ e $\left.\theta_{z}\right)$ nodais nas direções $\mathrm{X}, \mathrm{Y}$ e Z, que corresponde ao SHELL63 no software ANSYS.

Dois modelos numéricos da laje de concreto, um na condição não danificada e outro na danificada, foram implementados através do MEF no ANSYS.

A região danificada da laje apresenta dimensões iguais a $0,25 \mathrm{~m} \mathrm{x} 0,25 \mathrm{~m}$, com centro geométrico localizado nas coordenadas globais $X=2,625 \mathrm{~m} \mathrm{e} \mathrm{Z}=1,625 \mathrm{~m}$, tal como ilustrado na figura $1(\mathrm{a})$ e (b).

O número de gdl totais em ambos os modelos numéricos foi de 5022 gdl e a malha de elementos finitos obtida na condição danificada é ilustrada na figura 1(b). As condições de contorno implementadas em ambos os modelos numéricos são ilustradas na figura 1(a).

Figura 1 - Geometria e condições de contorno da laje de concreto (a) discretizada via MEF (b) com indicação da posição da área danificada.

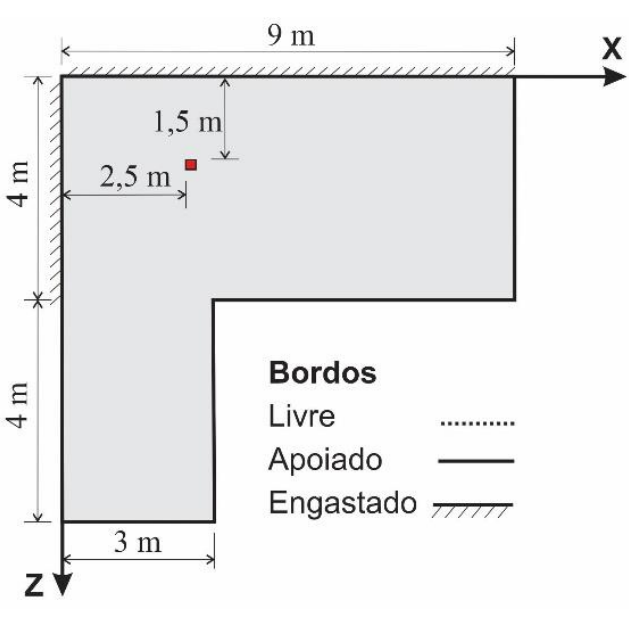

(a)

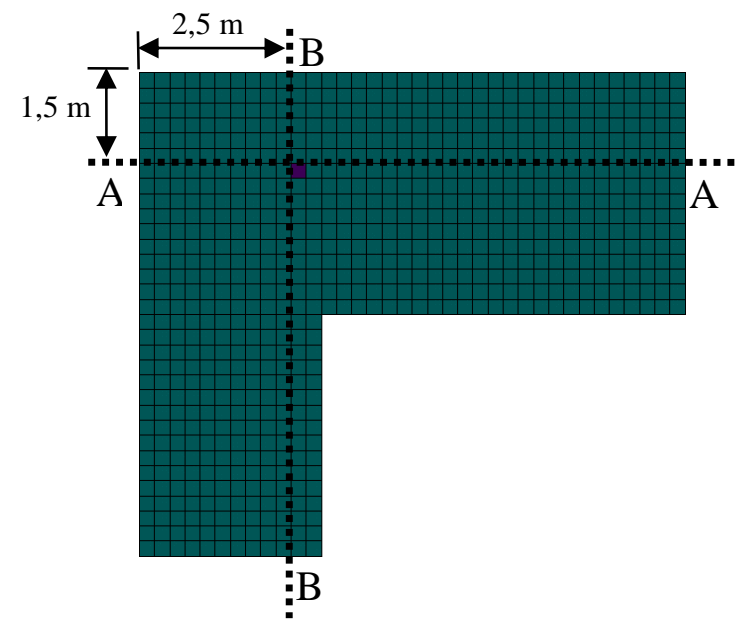

(b)

A figural 2, ilustra o campo de deslocamentos verticais, perpendicular ao plano da laje (direção Y), obtida em ambos os modelos numéricos. Na figura $2 \mathrm{~b}$ o campo de deslocamentos verticais ilustrado foi obtido adotando $D$ igual a 0,20 para o material da região danificada, ou seja, correspondente a uma perda de $20 \%$ do módulo de Young $(E)$.

Figura 2 - Campo de deslocamentos verticais, em metros, ao longo do plano da laje de concreto nas condições não danificada (a) e danificada (b).

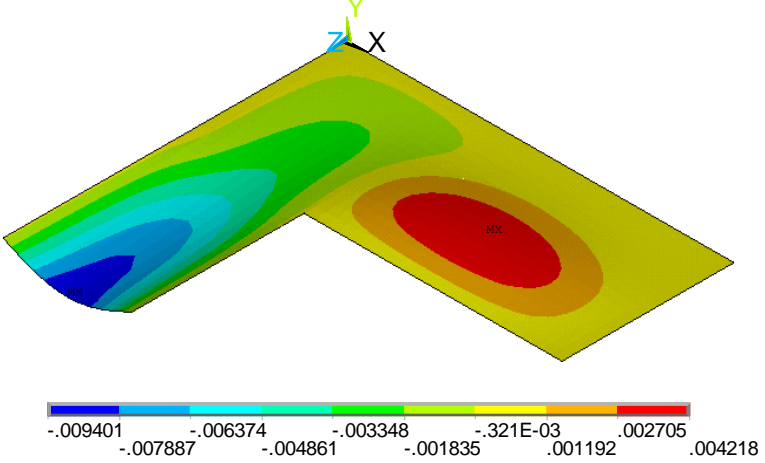

(a)

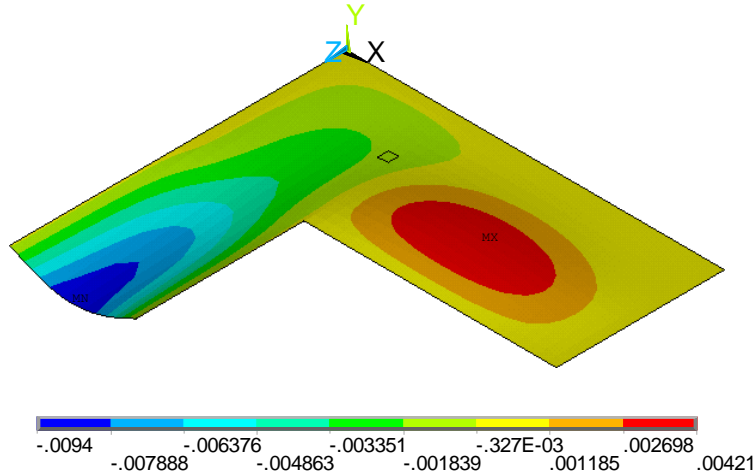

(b) 
Como pode ser observado na figura 2(a) e (b), em ambos os modelos numéricos o campo de deslocamentos verticais da laje de concreto é similar, não podendo distinguir visualmente a posição da região danificada.

O sinal da deflexão vertical obtido em ambos os modelos de laje, ao longo das direções A-A e B-B ilustradas na figura 1(b), é plotado na figura 3.

Figura 3 - Sinal da deflexão vertical obtido nas direções A-A (a) e B-B (b) da laje de concreto nas condições não danificada e danificada.

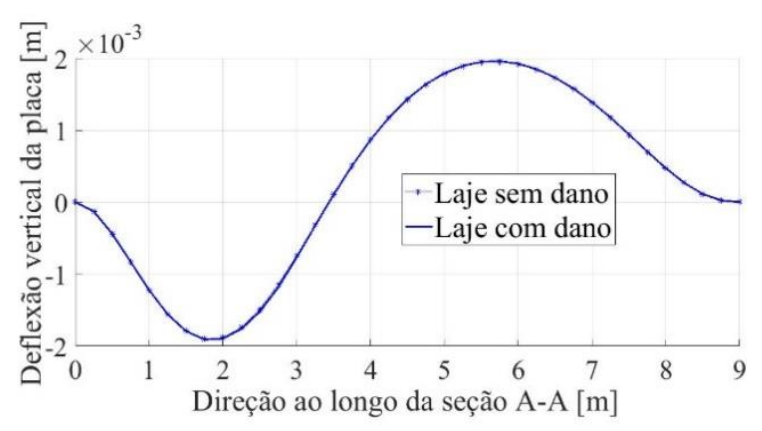

(a)

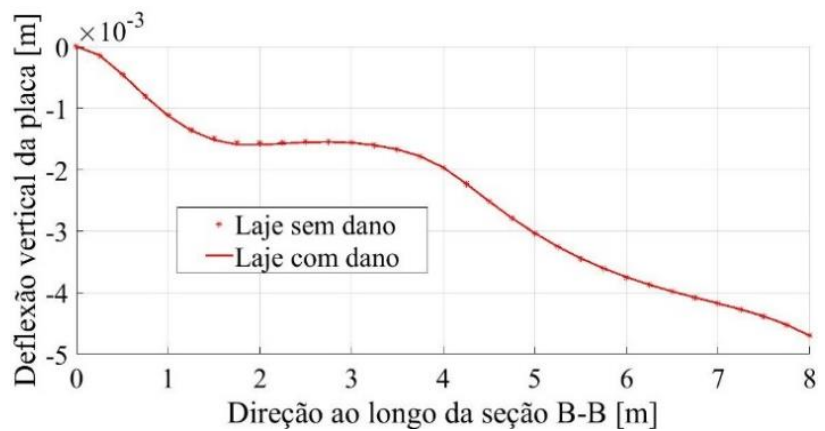

(b)

Pode-se observar visualmente na figura 3, e em ambas as direções A-A (figura 3(a)) e B-B (figura 3(b)) da laje, que o deslocamento vertical nas situações danificada e não danificada é praticamente o mesmo, havendo trechos com sobreposição entre os dois gráficos.

Após o uso das técnicas de interpolação cúbica e de regularização de Tikhonov, os sinais de deflexão ilustrados na figura 3 foram transformados via TDW pela wavelet mãe Daubechies (TDW-db), empregando dois níveis de decomposição. A técnica de interpolação adota um incremento de interpolação $\Delta x$ igual a 0,041 , obtendo-se assim dois novos vetores $s$ de dimensões 800 e 900, ou seja, proporcionais as dimensões da laje ao longo dos eixos X e Z (que são iguais a 8 e $9 \mathrm{~m}$ ), facilitando assim a leitura (nos gráficos dos $\mathrm{CW}$ ) das coordenadas $(\mathrm{X}, \mathrm{Z})$ do centro geométrico da área danificada.

As figuras 4, 5, 7 e 8 ilustram o uso da db10 no sinal obtido nas condições não danificada e danificada, nas direções A-A e B-B, e adotando $D$ igual a 0,20. Já as figuras 6 e 9 ilustram o sinal da diferença obtido entre as condições não danificada e danificada, respectivamente ao longo das direções A-A e B-B da laje. As figuras 4, 5, 7 e 8 utilizam $\beta_{j}=10^{6}$ enquanto as figuras 6 e 9 adotam $\beta_{j}=10^{10}$.

Figura 4 - CW da TDW-db10 aplicada ao sinal da estrutura sem dano ao longo da direção A-A

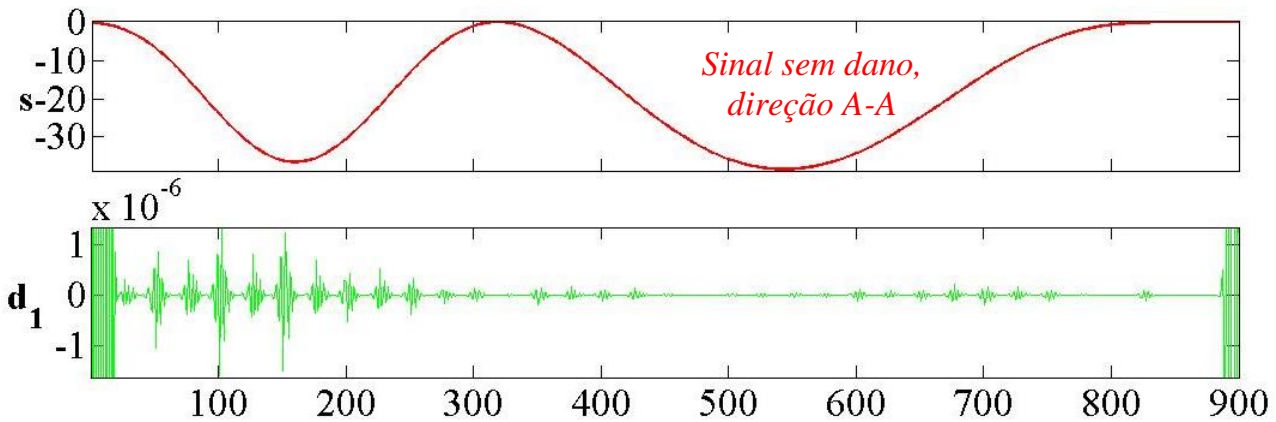


Campos; P. J. T.; Koroishi, E. H.; Faria, A. W.; Monitoramento da integridade estrutural de uma laje de concreto utilizando transformadas de wavelet. E\&S - Engineering and Science, 2020, 10:1.

Figura 5 - CW da TDW-db10 aplicada ao sinal da estrutura com dano ao longo da direção A-A.

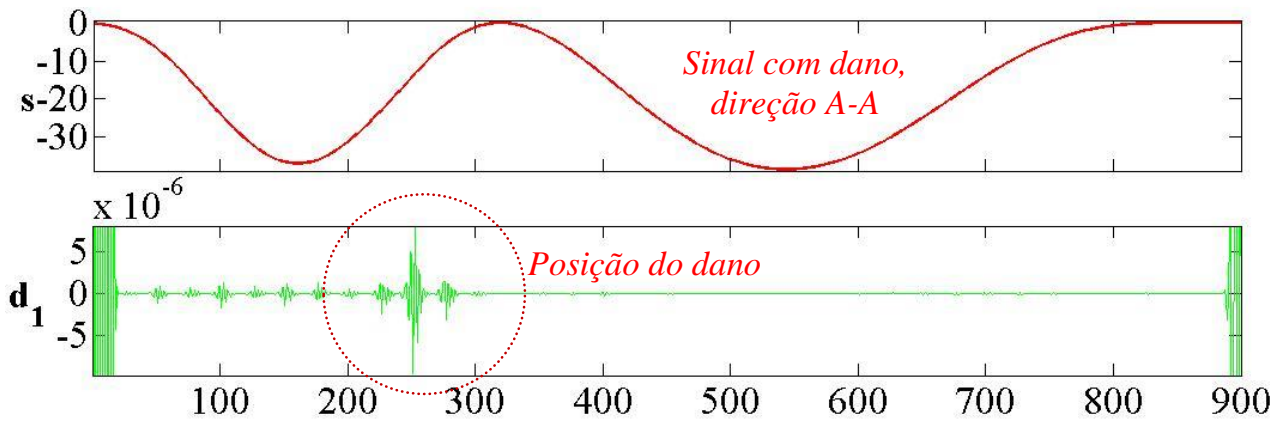

Figura 6 - CW da TDW-db10 aplicada ao sinal da diferença entre as condições com dano e sem dano ao longo da direção A-A.
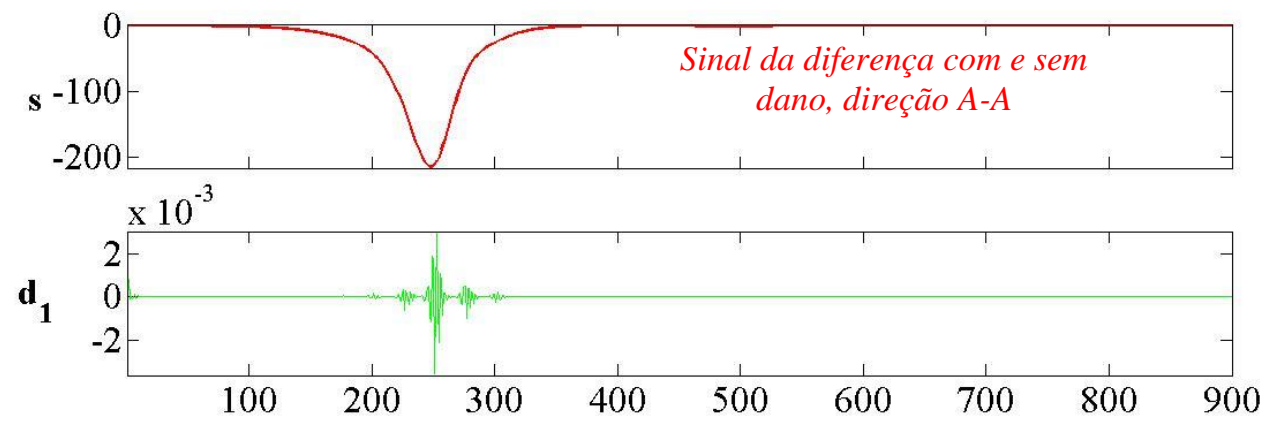

Figura 7 - CW da TDW-db10 aplicada ao sinal da estrutura sem dano ao longo da direção B-B.
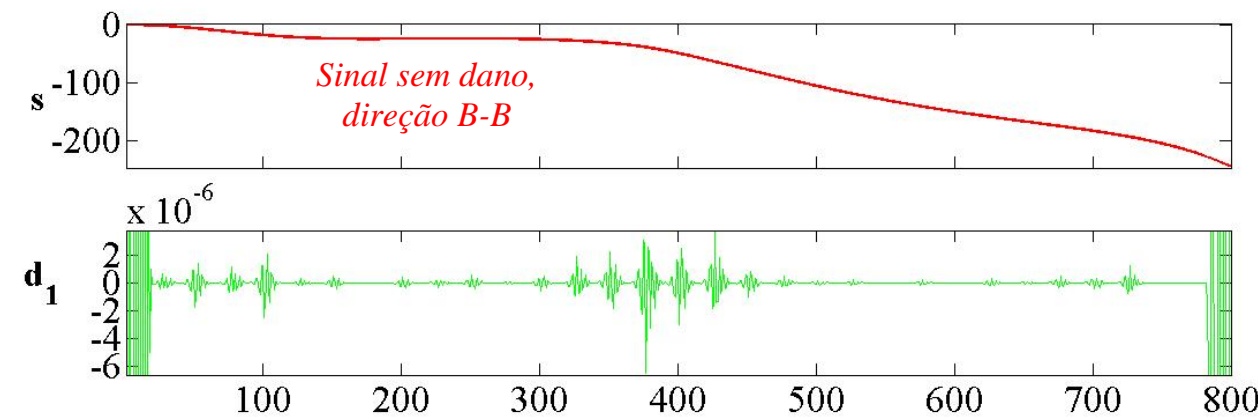

Figura 8 - CW da TDW-db10 aplicada ao sinal da estrutura com dano ao longo da direção B-B.
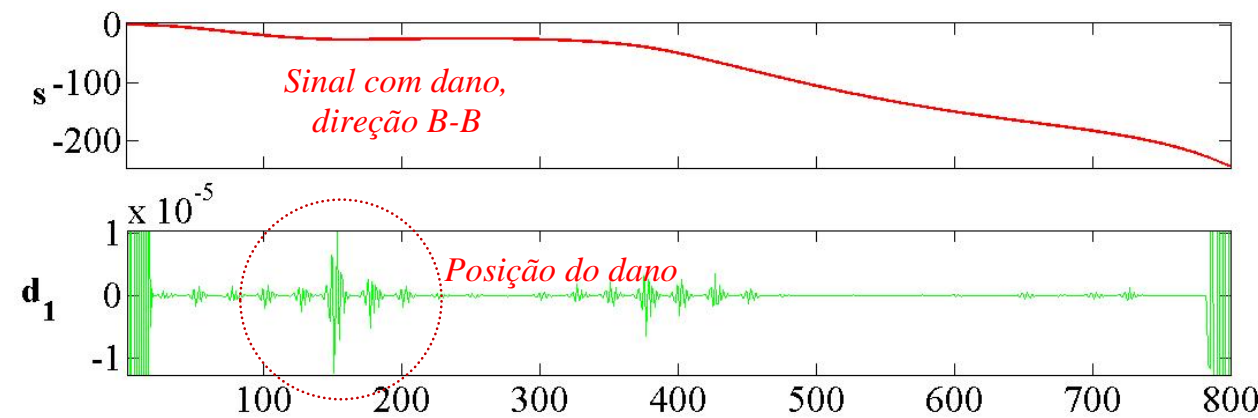
Campos; P. J. T.; Koroishi, E. H.; Faria, A. W.; Monitoramento da integridade estrutural de uma laje de concreto utilizando transformadas de wavelet. E\&S - Engineering and Science, 2020, 10:1.

Figura 9 - CW da TDW-db10 aplicada ao sinal da diferença entre as condições com dano e sem dano ao longo da direção B-B.

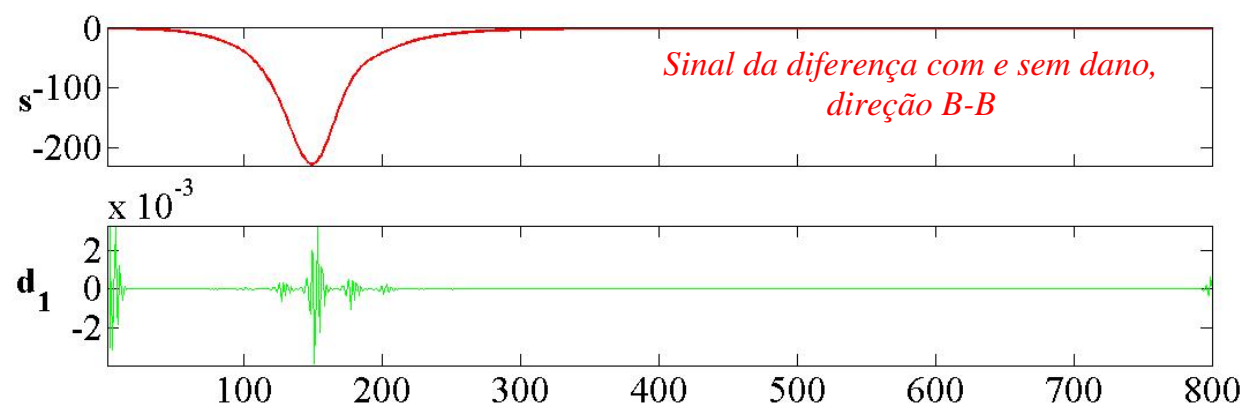

A localização do dano nas figuras de 4 a 9 é dada quando os CW dos gráficos ilustrados assumem picos de máxima amplitude. Nessas figuras $\mathbf{s}$ é a amplitude do sinal da deflexão vertical obtida e $\mathbf{d}_{\mathbf{1}}$ são os CW correspondentes ao segundo nível de decomposição da TWD$\mathrm{db} 10$.

A reconstrução do sinal $\mathbf{s}$ com dois níveis de decomposição pode ser dada pela expressão: $\mathbf{s}=\mathbf{a}_{2}+\mathbf{d}_{\mathbf{2}}+\mathbf{d}_{\mathbf{1}}$, sendo $\mathbf{a}_{2}$ um coeficiente de aproximação (sinal de baixa frequência que contém menos ruído se comparado ao sinal $\mathbf{s}$ original) da decomposição do sinal $\mathbf{s}$, e d2 trata-se de um coeficiente de detalhe (sinal de alta frequência e que contém a maior parte dos ruídos do sinal original).

Observa-se nas figuras 5 e 8 que, em ambas as direções analisadas na laje, não foi preciso conhecer o sinal da laje na condição não danificada para localizar o dano. Pode-se observar também nas figuras 6 e 9 que o sinal da diferença entre as condições não danificada e danificada consegue captar a localização do dano na laje de concreto, quando os CW atingem o seu pico de máximo.

A simulação posterior procura ilustrar a capacidade da TDW de localizar a área danifica utilizando diferentes valores de $D(0,10,0,15,0,20,0,60$ ou 0,90$)$, utilizando somente o sinal da laje na condição danificada. As figuras 10 e 11 ilustram os valores dos $\mathrm{CW}$, obtidos respectivamente nas direções A-A e B-B da laje empregando a wavelet mãe sym7 (em 2 níveis de decomposição) com esses diferentes valores de $D$.

Pode-se observar na figura 10 , para valores de dano $D$ menores que 0,20 , que a transformada sym7 não consegue captar com clareza a posição da área danificada. Pode-se também constatar através dessa mesma figura a existência de uma pequena perturbação à esquerda nos valores dos $\mathrm{CW}$ em virtude da proximidade do engaste da laje ao longo do eixo $\mathrm{Z}$ (posição $X=0$ ). 
Campos; P. J. T.; Koroishi, E. H.; Faria, A. W.; Monitoramento da integridade estrutural de uma laje de concreto utilizando transformadas de wavelet. E\&S - Engineering and Science, 2020, 10:1.

Figura 10 - CW da TDW-sym7 empregada ao sinal obtido na direção A-A da laje de concreto para diferentes valores de variável escalar $D$.
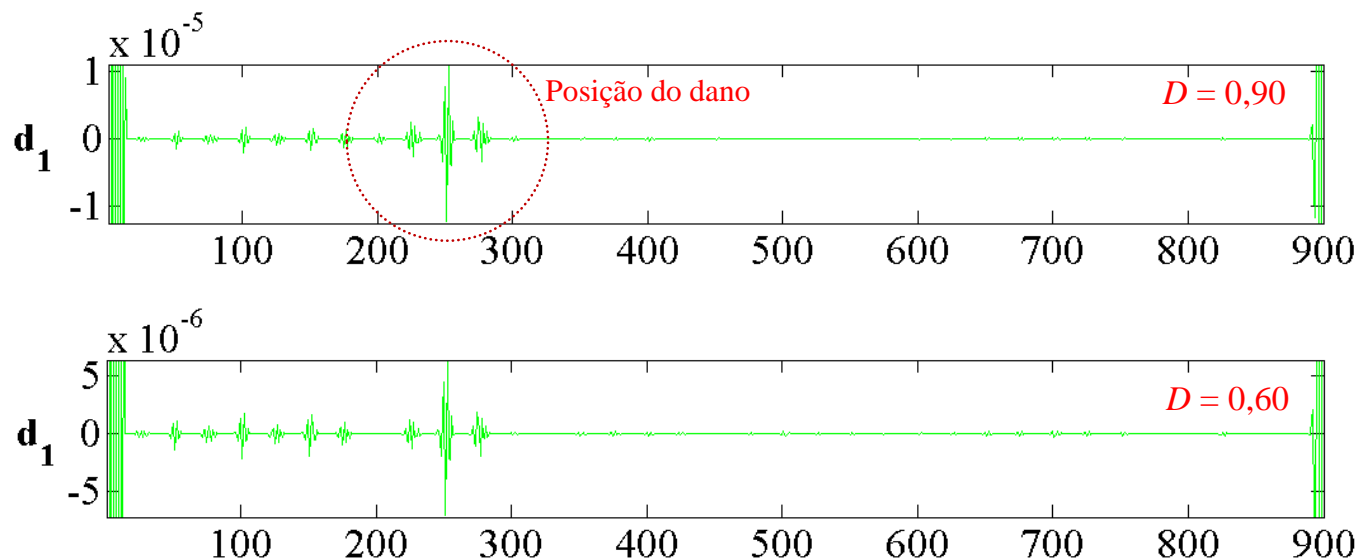

(b)

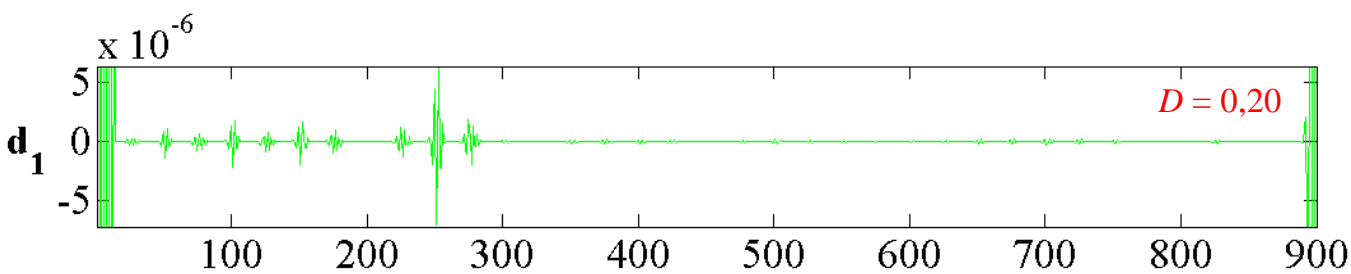

(c)

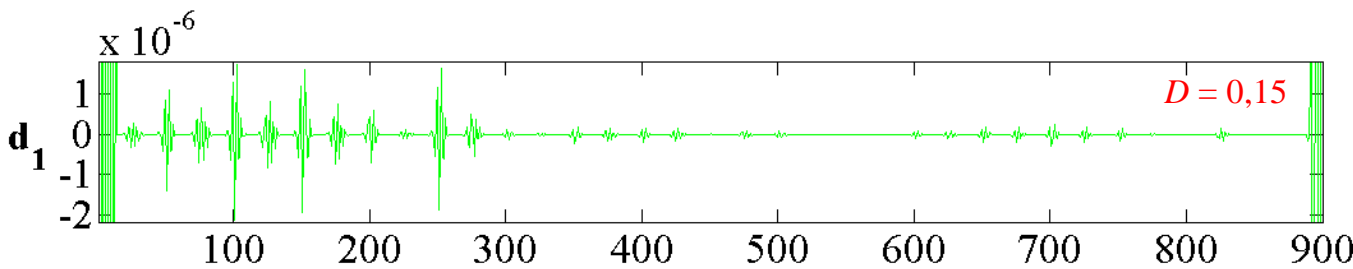

(d)

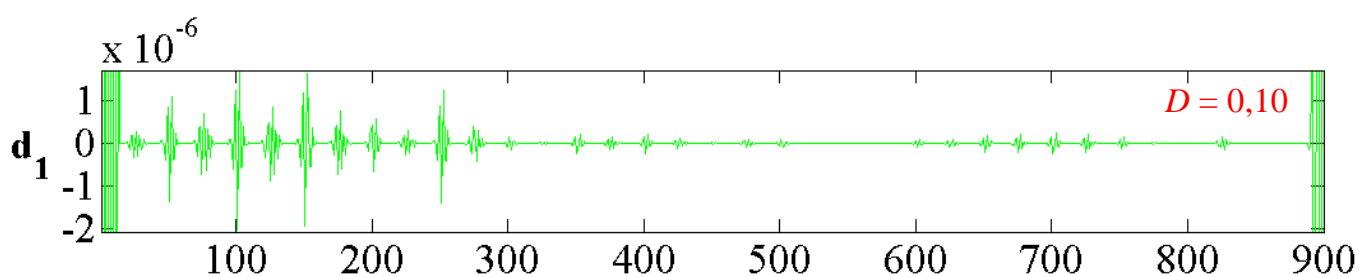

(e)

A figura 11 ilustra que quando $D$ é menor que 0,20 a wavelet sym 7 não consegue captar com clareza a posição do dano. Além disso, na posição 400 (na região central do gráfico dos $\mathrm{CW}$ ) pode ser observada uma interferência no sinal $\mathbf{s}$ devido as condições de contorno oriundas do bordo apoiado, paralelo a direção $\mathrm{X}$ e localizado em $\mathrm{Z}=4 \mathrm{~m}$. Além disso, também existe uma pequena perturbação à esquerda do gráfico dos $\mathrm{CW}$ oriunda do bordo apoiado ao longo do eixo Z. Portanto, ambas as perturbações oriundas das condições de contorno impedem a localização de maneira clara da posição do dano quando a variável escalar de dano é menor ou igual a 0,20 . 
Campos; P. J. T.; Koroishi, E. H.; Faria, A. W.; Monitoramento da integridade estrutural de uma laje de concreto utilizando transformadas de wavelet. E\&S - Engineering and Science, 2020, 10:1.

Figura 11 - CW da TDW-sym7 empregada ao sinal obtido na direção B-B da laje de concreto para diferentes valores de variável escalar $D$.

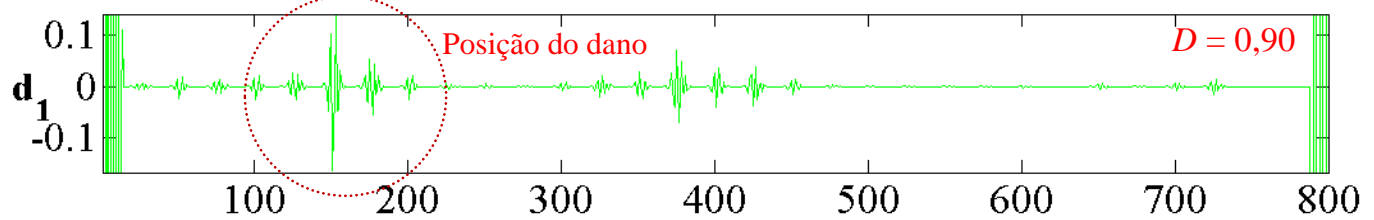

(a)

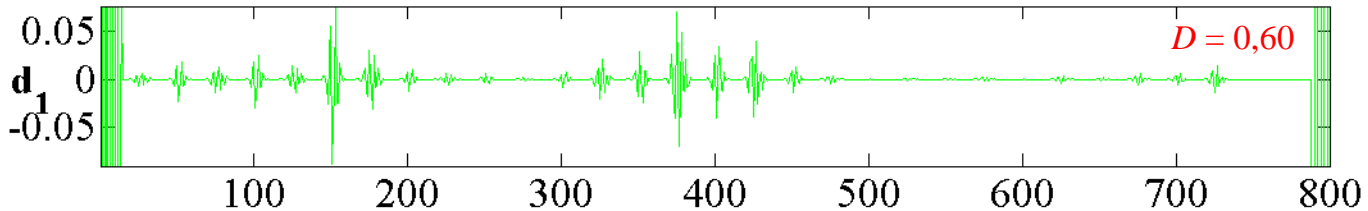

(b)

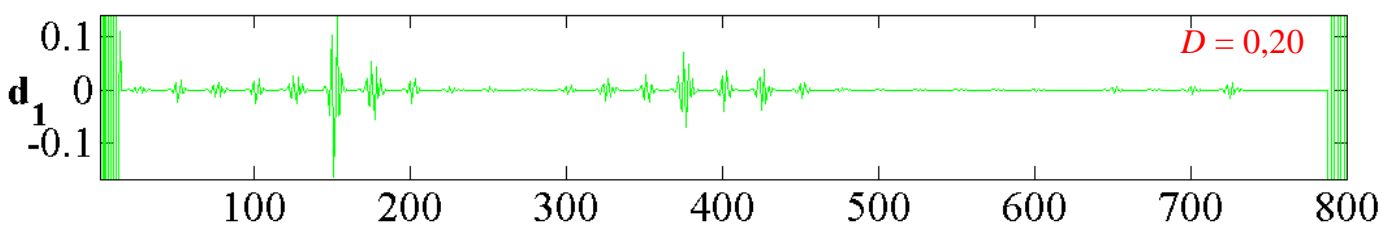

(c)
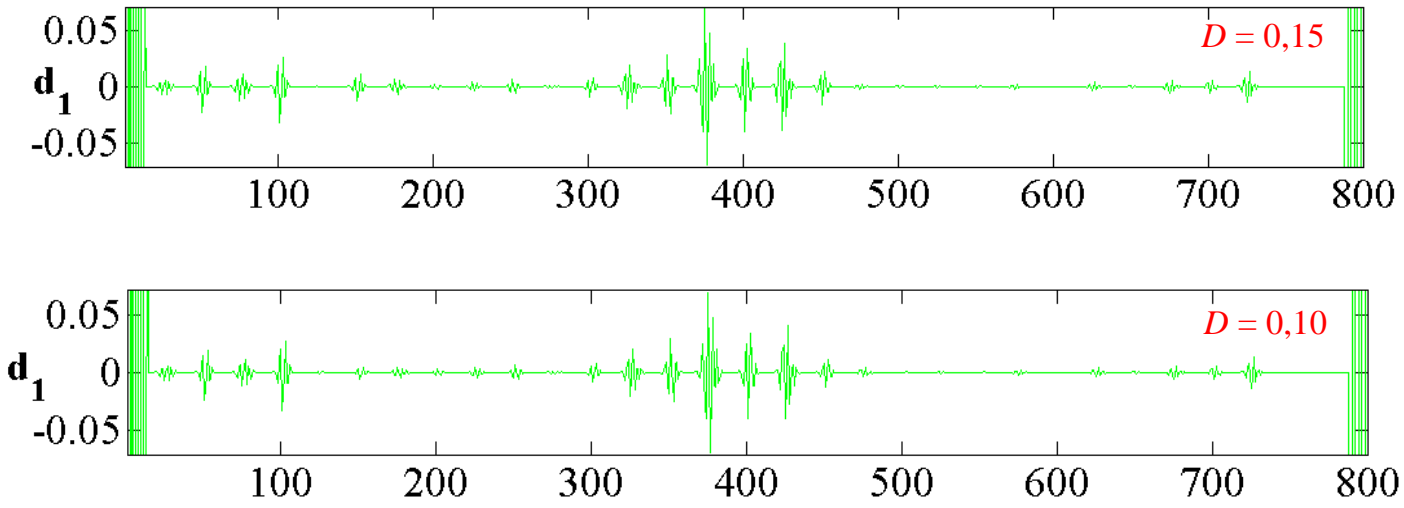

(e)

A figura 12 ilustra o estudo da influência da variação dos parâmetros $\beta_{j}$ da regularização de Tikhonov, adotando: $\beta_{0}=\beta_{1}=\beta_{2}$, nos CW obtidos com a TDW rbior3.5 (empregando 1 nível de decomposição, ou seja: $\mathbf{s}=\mathbf{a}_{\mathbf{1}}+\mathbf{d}_{\mathbf{1}}$ ) aplicada ao sinal $\mathbf{s}$ obtido ao longo da direção AA da laje na condição danificada (adotando $D=0,20$ ).

Como pode ser observado na figura 12 , que somente valores de $\beta_{j}$ maiores ou iguais a 100 ilustram com clareza a localização do centro geométrico da região danificada na laje. Podese notar também nessa mesma figura uma pequena perturbação, à sua esquerda, em virtude da proximidade do apoio, do tipo engaste, da laje de concreto (localizado ao longo do eixo $\mathrm{Z}$ ). 
Campos; P. J. T.; Koroishi, E. H.; Faria, A. W.; Monitoramento da integridade estrutural de uma laje de concreto utilizando transformadas de wavelet. E\&S - Engineering and Science, 2020, 10:1.

Figura 12 - Influência dos parâmetros $\beta_{j}$ nos valores do CW da transformada rbior3.5 aplicada ao sinal da deflexão da laje segundo a direção A-A.
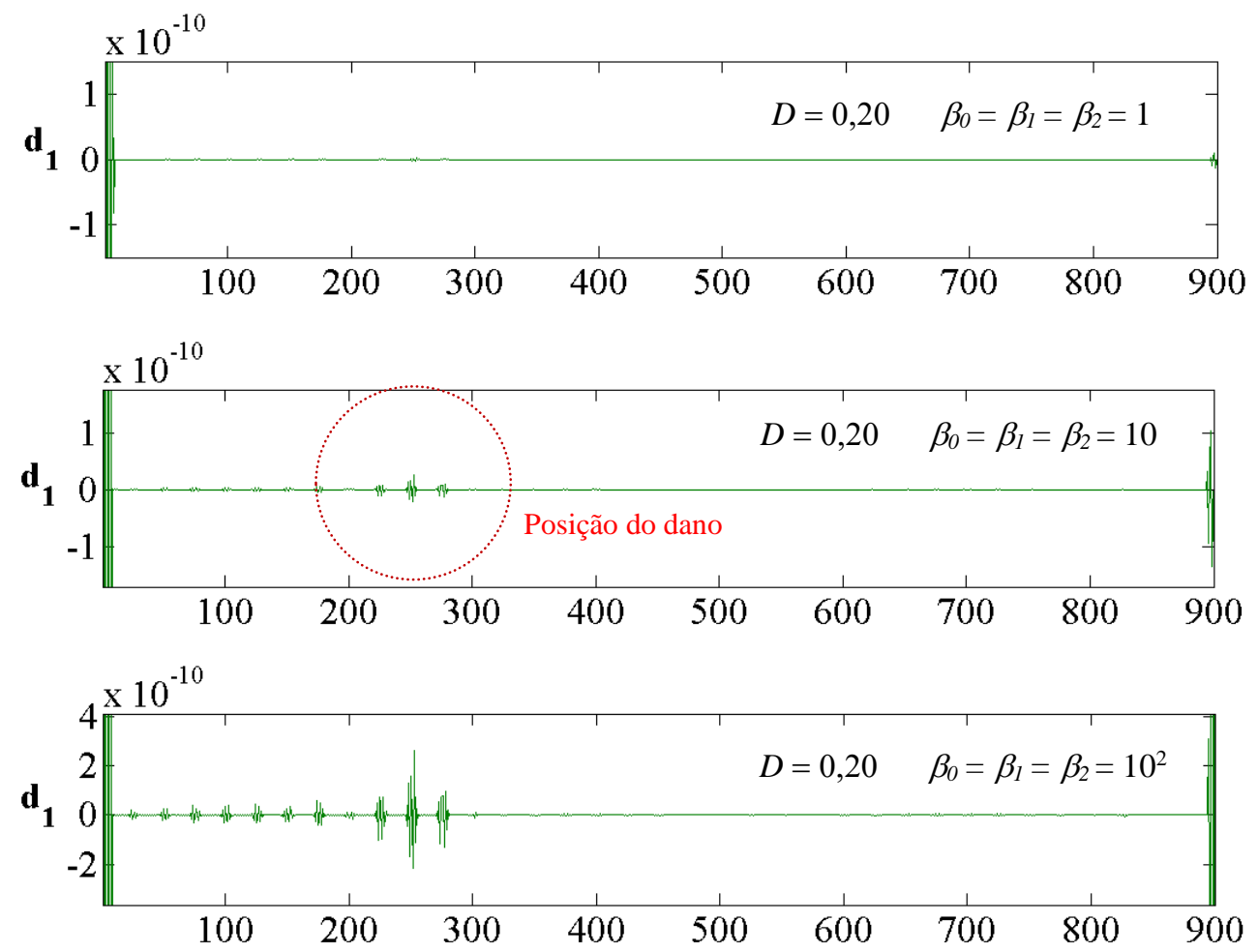

(c)
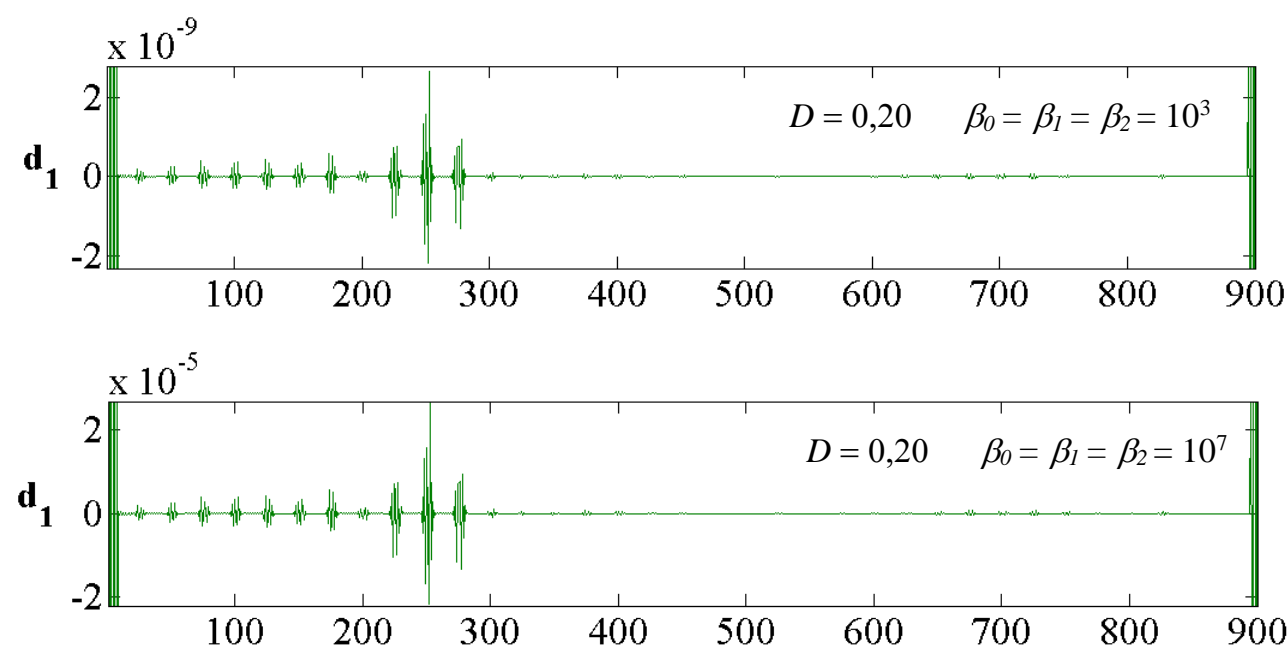

(e)

\section{CONCLUSÕES}

A TWD empregando as wavelets mães db, sym e rbior foi capaz de identificar a posição do centro geométrico da região danificada na laje de concreto, cujo dano é dado na forma de perda de rigidez localizada, no entanto, para valores da variável escalar de dano menores que $0,20(D<0,20)$, que corresponde a um diminuição de $20 \%$ do módulo de Young do concreto, não foi possível localizar o dano sem conhecer também o sinal da laje na condição não danificada, através do uso do sinal da diferença entre as condições não danificada e danificada. Para os demais casos, ou seja, danos com magnitude maior ou igual a $0,20(D \geq 0,20)$, somente é necessário conhecer o sinal da estrutura na condição danificada.

As condições de contorno aplicadas na estrutura e sua mudança de rigidez conforme a direção analisada, por ser uma da laje em "L", podem amplificar os valores dos CW e assim 
Campos; P. J. T.; Koroishi, E. H.; Faria, A. W.; Monitoramento da integridade estrutural de uma laje de concreto utilizando transformadas de wavelet. E\&S - Engineering and Science, 2020, 10:1.

conduzir a interpretações errôneas da localização do dano. Para limitar esse tipo de interferência na interpretação dos gráficos dos $\mathrm{CW}$, sugere-se a obtenção do sinal da diferença entre os sinais obtidos nas situações sem e com dano.

A escolha adequada do parâmetro $\beta_{j}$ da regularização de Tiknokov mostrou-se importante na identificação da região danificada, sendo que valores maiores ou iguais a 100 mostraram-se os mais adequados.

\section{REFERÊNCIAS}

ADDISON, P. S. The Ilustrated Wavelet Transform Handbook: Introductory Theory and Applications in Science, Enginnering, Medicine and Finance. $2^{\text {nd }}$ ed. Scotland: CRC Press, 2017.

ABNT - ASSOCIAÇÃO BRASILEIRA DE NORMAS TÉCNICAS. NBR 6118. Projeto de estruturas de concreto - Procedimento. RJ, Associação Brasileira de Normas Técnicas, 2014.

ABNT - ASSOCIAÇÃO BRASILEIRA DE NORMAS TÉCNICAS. NBR 9607. Prova de carga em estrutura de concreto e protendido: procedimento. RJ, Associação Brasileira de Normas Técnicas, 1986.

BATHE, K-J. Finite element procedures. $2^{\text {nd }}$ Edition. Watertown: Prentice-Hall, 2014.

BECK, J.V., BLACKWELL, B., ST. CLAIR JR, C. R. Inverse Heat Conduction. III-Posed Problems, New York: J. Wiley \& Sons, 1985.

CAMACHO, J. S. Concreto Armado: Estados Limites de Utilização. Apostila de Curso UNESP - Ilha Solteira/SP. 2015.

CHAPRA, S. C. Métodos numéricos aplicados com MATLAB para engenheiros e cientistas. Editora AMGH, 2013.

COSTA SILVA, R. S. Y. Monitoramento e identificação numérico e experimental de danos em vigas e pontes de aço e concreto. Tese de Doutorado apresentada a Universidade de Brasília - UNB - Brasília/DF. 2015.

FARIA, A. W., SILVA, R. A., KOROISHI, E. H. Matrix Damage Detection in Laminated Composite Structures by Discrete and Continuous Wavelet transforms using Vibrations Modes. Journal of Aerospace Technology and Management, v. 9, p. 431-441, 2017. dx.doi.org/10.5028/jatm.v9i4.804

GALVÃO, L. C., NUNES, L. F. Cálculo Numérico. Apostila de Curso - UFTPR. 2021.

HECKE M. B. Métodos dos Elementos Finitos Aplicados à Engenharia de Estruturas: Lajes com Geometrias Espaciais. Apostila de Curso - UFPR - Curitiba/PR. 2012.

JANELIUKSTIS, R., RUCEVSKIS, S., AKISHIN, P., CHATE, A. Wavelet transform based damage detection in a plate structure. In: WORLD MULTIDISCIPLINARY CIVIL ENGINEERING-ARCHITECTURE-URBAN PLANNING SYMPOSIUM, p. 127-132, 2016, Riga, Latvia. Riga: WMCAUS, 2016. 
MAIO, C. E. B. Técnicas para Monitoramento de Integridade Estrutural Usando Sensores e Atuadores Piezoelétricos. Dissertação de Mestrado apresentada a Escola de Engenharia de São Carlos - USP - São Carlos/SP. 2011.

OVANESOVA, A. V., SUÁREZ, L. E. Applications of Wavelet Transforms to Damage Detection in Frame Structures. Engineering Structures, v. 26, p. 39-49, 2004. doi.org/10.1016/j.engstruct.2003.08.009

STARK, H. G. Wavelets and Signal Processing an Application-Based Introduction. New York: Springer, 2005.

YAN, Y. J., YAM, L. H. Online Detection of Crack Damage in Composite Plates using Embedded Piezoelectric Actuators/Sensors and Wavelet Analysis. Composite Structures, v. 58, p. 29-38, 2002. doi.org/10.1016/S0263-8223(02)00043-0

YANG, J. M., YANG, Z. W., TSENG, C. M. Damage Detection in Stiffened Plates by Wavelet Transform. International Journal of Naval Architecture and Ocean Engineering, v. 3, p. 126-135, 2011. https://doi.org/10.2478/IJNAOE-2013-0055 